\title{
La pertinence de la Global Health pour la Suisse
}

\section{Nicolaus Lorenz, Bettina Borisch, Michaela Told, John-Paul Vader}

1 Basch P E. Textbook of International Health. Oxford Univ Pr.1999.

\section{Correspondance:}

Nicolaus Lorenz, executive MBA, MPH, MD Swiss Tropical and Public Health Institute Socinstrasse 59 Boîte postale CH-4002 Bâle Tél. 0612848125 Fax 0612848103
Si la Global Health (santé globale) peut sembler n'être qu'un énième nouveau terme à la mode, il reste toutefois d'une importance primordiale pour la Suisse. Du point de vue helvétique, la «Global Health» paraît être une contrée vaste, lointaine et exotique: difficile à atteindre, bizarrement attirante, peut-être un peu dangereuse, mais toutefois offrant la perspective qu'un tel objectif pourrait être bénéfique et gratifiant [1]. C'est justement pour cela qu'il correspond très bien au contexte suisse, un pays dont la population compte parmi les plus grands voyageurs de ce monde. Les voyages sont extrêmement enrichissants, ils ouvrent à d'autres cultures ainsi qu'à des façons de voir et d'agir différentes. En médecine clinique, nous avons également l'habitude de regarder au delà de nos frontières et de bénéficier des expériences et résultats récoltés à travers le monde, bien que parfois l'entrée en pratique clinique prenne du temps.

En santé publique, l'importance de la dimension globale est reconnue depuis longtemps. Même si la récente pandémie H1N1 n'a pas atteint l'importance prédite par les professionnels, elle demeure un exemple de la vitesse à laquelle une pandémie peut se propager autour du globe.

Tous les pays doivent trouver des réponses aux difficultés de leurs propres systèmes de santé. Mais la Suisse peut aussi apprendre des échecs et des succès d'autre pays - et vice-versa. La campagne de prévention du VIH et Sida en Suisse a été largement reconnue et a influencé d'autres campagnes, par exemple.

L'importance du savoir helvétique en matière de santé publique se reflète dans les 18 Centres Collaborateurs de l'OMS (http://apps.who.int/whocc/) en Suisse. Ces centres d'excellence ont pour mission d'épauler l'OMS dans différents domaines spécifiques. En comparaison aux 33 centres installés en Allemagne, la Suisse en compte donc plus de la moitié que son voisin du nord et seulement quatre de moins que la France (22). La Suisse bénéficie également de structures uniques en Europe, telles que le Graduate Insitute à Genève, qui traite de la diplomatie globale en matière de santé. Aux côtes de l'OMS, beaucoup d'initiatives pour la santé globale ont choisi Genève, la capitale mondiale de la santé: les organisations et institutions suisses, comme l'Institut Tropical et de Santé Publique Suisse, collaborent étroitement avec le Fonds mondial de lutte contre le Sida, la tuberculose et le paludisme, et l'initiative
Médicaments contre les Maladies Négligées (DNDi). Santé Publique Suisse et l'Institut universitaire de Médecine Sociale et Préventive hébergent le secrétariat genevois de la Fédération Mondiale des Associations de Santé Publique.

Le groupe spécialisé «Global Health» a été créé au sein de la société de discipline médicale Santé Publique Suisse en vue de capitaliser les vastes expériences acquises dans ce domaine et de bénéficier de l'expérience internationale en Suisse.

\section{La Suisse peut aussi apprendre des échecs et des succès d'autre pays}

L'une des premières activités du groupe a été de formuler une définition appropriée au contexte helvétique du terme de Global Health. Après avoir pris connaissance de nombreux textes et de différents points de vue à ce sujet, nous aimerions proposer la définition suivante: la Global Health est un espace destiné à la recherche, à la pratique et aux règles y afférents, qui met la priorité sur l'amélioration de la santé, en général, et sur un accès équitable à la santé pour tous, en particulier. La Global Health répond aux questions de santé transnationales et s'intéresse aux déterminants et aux possibilités de solution. Elle implique beaucoup de disciplines, tant du secteur médical que d'autres domaines, promouvant ainsi la collaboration interdisciplinaire; elle encourage la bonne gouvernance pour anticiper et s'adapter à un environnement qui change rapidement.

A l'avenir, une plateforme sera créée dans le but d'offrir un accès aux expériences internationales et de les fructifier en Suisse mais aussi de partager les expériences acquises ici localement, avec des partenaires internationaux tels que les associations européenne et mondiale de santé publique.

Last but not least, le groupe spécialisé susmentionné va plaider pour une compréhension large et commune de la Global Health. Les personnes intéressées sont invitées à se joindre à ce travail (www. public-health.ch/). 